\title{
Wide Energy Bandwidth Superconducting Accelerating Cavities
}

\author{
Ji Qiang \\ Lawrence Berkeley National Laboratory \\ 1 Cyclotron Road, Berkeley, CA 94720, USA
}

\begin{abstract}
Superconducting cavities have been widely used in high intensity proton accelerators. In this paper, we discuss parameters that affect energy bandwidth of the accelerating cavity and propose a new energy averaged transit time factor to help choose transition energy between different sections and cavity geometry parameters in the linac design. These wide energy bandwidth superconducting cavities can potentially be used to accelerate a proton beam multiple times. A time-pass condition is defined to attain fixed RF phases during multiple passes of the beam. Such a superconducting recirculating proton linac could significantly reduce the number of $\mathrm{RF}$ cavities in the accelerator and lower construction and operational costs of the facility.
\end{abstract}

Keywords: superconducting cavities, recirculating proton linac

\section{Introduction}

Superconducting cavities have been widely used/proposed in high power proton $\left(H^{+} / H^{-}\right)$accelerators. A number of spallation neutron sources driven by high power proton accelerator were built and are under construction around the 5 world [1, 2, 3, 3, 4, 5]. It was also proposed as a driver for nuclear waste transmutation in subcritical nuclear power plant [6, 7, 8, 9], for production of tritium [10],

\footnotetext{
${ }^{*}$ Corresponding author

Email address: jqiang@lbl.gov (Ji Qiang)
}

Preprint submitted to Journal of $L^{A} T_{E} X$ Templates

May 23, 2015 
and for high intensity neutrino physics study [11, 12, 13]. Superconducting cavities were used/proposed in the main section of all of those facilities to accelerate the beam since those cavities can provide high accelerating gradient with little power loss on the wall. In addition, superconducting cavity also allows a larger cavity bore radius in comparison with the normal conducting cavity since the accelerating gradient of the cavity is not limited by the cavity bore radius. This helps reduce the potential proton beam losses, which is important for all high power accelerators in order to avoid the risk of radioactivity caused by the lost particles and to allow hand-on maintenance of the accelerator.

A single pass superconducting high power proton linear accelerator is architecturally simple but inefficient in the use of RF cavities compared with circular accelerators such as a synchrotron, where the beam passes through the same RF cavity many times. Moreover, building and operating superconducting cavities are expensive. To minimize the construction and the operational costs, it will be beneficial to keep the number and the type of RF cavities as low as possible. Table 1 gives a list of the superconducting cavities used/proposed in some high power $\mathrm{GeV}$ proton linear accelerators. It is seen that in those facilities, a few types of superconducting cavities were used for major energy gain. For exam${ }_{25}$ ple, in the Project-X accelerator design, six types of superconducting cavities were proposed to accelerate the beam from $2 \mathrm{MeV}$ to $8 \mathrm{GeV}$. A single type elliptical cavity was used to accelerate the beam from $177 \mathrm{MeV}$ to $480 \mathrm{MeV}$ kinetic energy, one type of cavity to accelerate the beam from $480 \mathrm{MeV}$ to 3 $\mathrm{GeV}$, and one type of cavity from $3 \mathrm{GeV}$ to $8 \mathrm{GeV}$. These energy ranges can each be divided into a much smaller energy range if one allows the beam to pass through the same cavity multiple times. This significantly saves the number of cavities needed in the accelerator and reduces the construction and the operational costs. In this study, we discuss parameters that affect the energy bandwidth of the superconducting cavity, the choice of the transition energy 35 between different sections of a linac to maximize the acceleration efficiency and to minimize the number of cavities, and a potential application of these wide energy bandwidth cavities to a $\mathrm{GeV}$ recirculating proton linac concept. 
Table 1: Superconducting cavities used/proposed in some $\mathrm{GeV} H^{+} / H^{-}$accelerators.

\begin{tabular}{|c|c|c|c|c|}
\hline Facility & Cavity type & Freq. (MHz) & $\beta_{g}$ & Energy $(\mathrm{MeV})$ \\
\hline \multirow[t]{2}{*}{ SNS [2] } & 6 cell-Ellipt. & 805 & 0.61 & $186-387$ \\
\hline & 6 cell-Ellipt. & 805 & 0.81 & $387-1000$ \\
\hline \multirow[t]{3}{*}{ ESS [5] } & $\mathrm{SR}$ & 352.2 & 0.50 & $90-216$ \\
\hline & 6 cell-Ellipt. & 704.4 & 0.67 & $216-571$ \\
\hline & 5 cell-Ellipt. & 704.4 & 0.86 & $571-2000$ \\
\hline \multirow[t]{6}{*}{ Project-X [11] } & HWR & 162.5 & 0.09 & $2-11$ \\
\hline & SSR & 325 & 0.19 & $11-38$ \\
\hline & SSR & 325 & 0.43 & $38-177$ \\
\hline & 5 cell-Ellipt. & 650 & 0.61 & $177-480$ \\
\hline & 5 cell-Ellipt. & 650 & 0.9 & $480-3000$ \\
\hline & 9 cell-Ellipt. & 1300 & 1.0 & $3000-8000$ \\
\hline \multirow[t]{2}{*}{ SPL [13] } & 5 cell-Ellipt. & 704.4 & 0.65 & $180-643$ \\
\hline & 5 cell-Ellipt. & 704.4 & 1.0 & $643-3500$ \\
\hline \multirow[t]{2}{*}{ APT [10] } & 5 cell-Ellipt. & 700 & 0.64 & $217-469$ \\
\hline & 5 cell-Ellipt. & 700 & 0.82 & $469-1700$ \\
\hline \multirow[t]{4}{*}{ C-ADS [7] } & SSR & 325 & 0.21 & $10-40$ \\
\hline & SSR & 325 & 0.40 & $40-160$ \\
\hline & 5 cell-Ellipt. & 650 & 0.63 & $160-360$ \\
\hline & 5 cell-Ellipt. & 650 & 0.82 & $360-1500$ \\
\hline \multirow[t]{3}{*}{ I-ADS [8] } & 5 cell-Ellipt. & 704.4 & 0.49 & $100-192$ \\
\hline & 5 cell-Ellipt. & 704.4 & 0.62 & $192-435$ \\
\hline & 5 cell-Ellipt. & 704.4 & 0.8 & $435-1014$ \\
\hline
\end{tabular}


Recirculating electron linac as an efficient accelerator has operated for many years [14, 15]. It provides a cost/performance optimum between the straight linear accelerator and the circular accelerator. It was also proposed in a number of next generation light sources [16, 17, 18, 19, 20]. A series of workshops were dedicated to this topic [21]. Besides accelerating electron beam, it was also proposed to accelerate muon beam for neutrino factory application [22]. In hadron accelerators, recirculating induction linac was proposed to accelerate ion beam for heavy fusion application [23]. To the best of our knowledge, up to now, there is no recirculating linac that was proposed to accelerate a proton beam. In an electron linac, a single type of RF cavity is sufficient to accelerate the beam through nearly the entire accelerator since the electron velocity does not change much any more when its kinetic energy is beyond a few $\mathrm{MeV}$. In a proton linac, a single type of cavity is not efficient due to the phase slippage between the proton beam and the RF field during the process of acceleration. Multiple types of cavities with different cavity cell lengths are used to match the velocity change of the proton with the RF accelerating field in order to attain a good acceleration efficiency. However, as shown in Table 1 and will also be discussed in the following section, by appropriately choosing RF cavity parameters, a single type of cavity can still cover a broad range of energy for a proton beam and meanwhile has a good acceleration efficiency.

The organization of the paper is as follows: in Section 2, we will discuss the choice of some superconducting cavity parameters to maximize its energy bandwidth and acceleration efficiency, and present a potential application to a $\mathrm{GeV}$ recirculating linac concept; in Section 3, we will discuss potential challenges in such a facility.

\section{Wide energy bandwidth superconducting cavities and a potential application}

The design of accelerator starts with the choice of accelerating cavity parameters. In an electron linac, a single type of cavity is sufficient to accelerate 
the beam from a few $\mathrm{MeV}$ to multiple $\mathrm{GeV}$, while in a proton linac, a number of types of cavities are needed to accelerate the beam to $\mathrm{GeV}$ with a good acceleration efficiency. A good acceleration efficiency is important in order to save the number of RF cavities needed in the accelerator. On the other hand, using too many types of RF cavities will increase the costs of designing and operating those cavities, especially for the superconducting cavities. The final choice of the RF cavity is a balance of acceleration efficiency and the type of cavities.

The acceleration efficiency can be measured by the transit time factor. For 75 a given RF cavity, the energy gain $\Delta E_{c}$ of a charged particle through the cavity can be written as:

$$
\Delta E_{c}=q V T \cos (\phi)
$$

where $q$ is the charge of the particle, $V=\int_{0}^{L}\left|E_{z}(0, z)\right| d z$ is the voltage across the cavity, $E_{z}$ is the longitudinal accelerating electric field on axis, $T$ is the transit time factor, $\phi$ is the design phase with respect to the maximum energy gain.

so From above equation, we can see that for a given design phase and voltage, in order to gain more energy, the transit time factor should be as large as possible. For a periodic RF cavity with harmonic distribution of electric field along the axis, i.e. $E_{z}=\sin (\omega z /(\beta c)) \exp (i \omega t)$, the normalized transit time factor $T_{0}$ can be given by [11, 12]:

$$
\begin{aligned}
T_{0}(\beta)= & \frac{2 \beta}{\pi n}\left(\frac{\sin \left(\pi n\left(\beta-\beta_{G}\right) /(2 \beta)\right)}{\beta-\beta_{G}}-\right. \\
& \left.(-1)^{n} \frac{\sin \left(\pi n\left(\beta+\beta_{G}\right) /(2 \beta)\right)}{\beta+\beta_{G}}\right)
\end{aligned}
$$

85 normalized particle velocity, $c$ is the speed of light in vacuum, $\beta_{G}$ is the geometry parameter that characterizes the synchronization between the particle and the RF field inside the cavity, and $T=\frac{\pi}{4} T_{0}$. For a $\pi$-mode cavity, the cell length in the cavity is $\frac{1}{2} \beta_{G} \lambda$, where $\lambda$ is the RF wavelength of the field inside the cavity. 90 The above equation gives a good approximation to the transient time factors obtained by numerical integration of actual time dependent electric field for the 


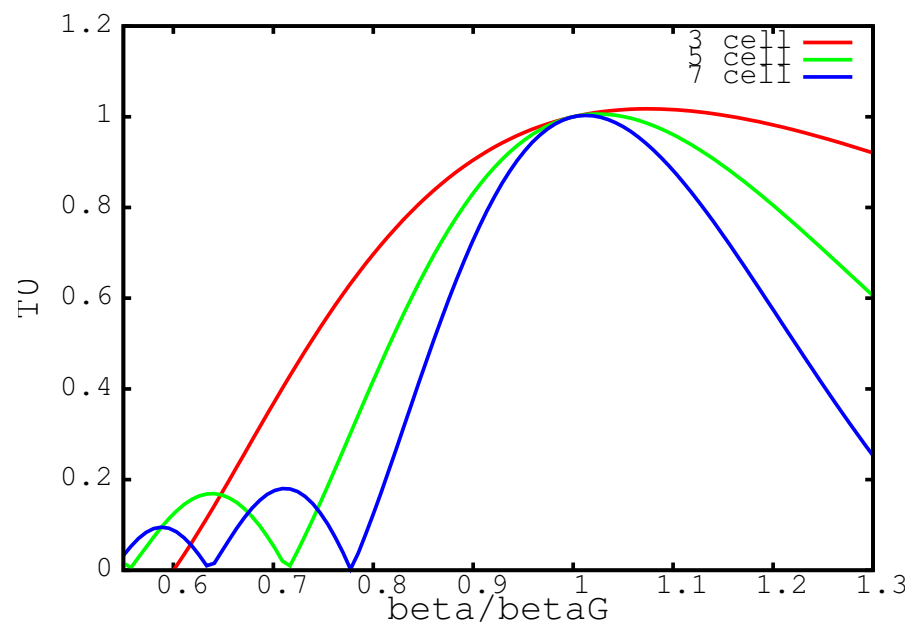

Figure 1: Normalized transit time factor as a function of the ratio of the particle beta to the geometric beta for different number of cells in a cavity.

Project-X cavities, which are the ones proposed to be used in this study. The transit time factor depends on the velocity of the proton inside the RF cavity and the cavity parameters. Figure 1 shows a plot of the transit time factor as a function of the ratio of the particle velocity $\beta$ to the cavity geometric $\beta_{G}$ with different number of cells per cavity. It is seen that as the number of cells per cavity increases, the velocity acceptance (the range of particle velocity to attain a good acceleration efficiency) or energy bandwidth decreases. A small number of cells per cavity, e.g. $n=3$, provides a large velocity acceptance. However, for a given energy range, this may require a use of a large number of RF cavities, lower real estate accelerating gradient, and an increase the system complexity. If the number of cells per cavity is too large, besides the decrease of the velocity acceptance, the fabrication of the cavity also becomes more challenge. As a compromise, in most accelerators listed in Table 1 , a five cell $(n=5)$ per cavity structure was used to accelerate the proton beam energy from the 100 $\mathrm{MeV}$ level to the $\mathrm{GeV}$ level. In this study, we also propose to use such a five-cell superconducting cavity with $650 \mathrm{MHz}$ frequency in the recirculating linac to accelerate the proton beam from $150 \mathrm{MeV}$ to multiple GeV. The 650 $\mathrm{MHz}$ superconducting cavity has been extensively studied under the Project- 

gradient $(>15 \mathrm{MV} / \mathrm{m})$ under continuous wave $(\mathrm{CW})$ operation and has also a large aperture size $(\sim 100 \mathrm{~mm})$ for beam pass.

Figure 2 shows a schematic plot of a potential application of above wide energy bandwidth superconducting cavities to a $\mathrm{GeV}$ recirculating proton linac. It consists of three sections with each section using a single type of the superconducting cavity. The first section accelerates the proton beam to a few hundred $\mathrm{MeV}$, the second section accelerates the beam to $2 \mathrm{GeV}$, and the last section accelerates the beam to multiple $\mathrm{GeV}$. The choice of $2 \mathrm{GeV}$ energy at the exit of the second section is out of the consideration that most high power accelerator driven systems will have a final beam energy below $2 \mathrm{GeV}[6]$. For the energy between $150 \mathrm{MeV}$ and $2 \mathrm{GeV}$, we will use two types of cavities with different geometric $\beta_{G}$. In order to determine the geometrical $\beta_{G}$ of those cavities and the transition energy between the two sections, we define an average transit time factor as:

$$
\bar{T}\left(\beta_{G}\right)=\frac{m c^{2}}{\Delta E_{\max }} \int_{\beta_{\text {in }}}^{\beta_{\text {out }}} \frac{T\left(\beta, \beta_{G}\right) \beta}{\left(1-\beta^{2}\right)^{3 / 2}} d \beta
$$

where $\Delta E_{\max }$ is the maximum energy gain through a section of cavities, $\beta_{\text {in }}$ is the normalized velocity at the entrance of the accelerator section, and $\beta_{\text {out }}$ is the normalized velocity at the exit of the section. The maximum energy gain through the section can be written as

$$
\Delta E_{\max }=q V N \bar{T}
$$

where $N$ is proportional to the number of cavities used. For a given range of

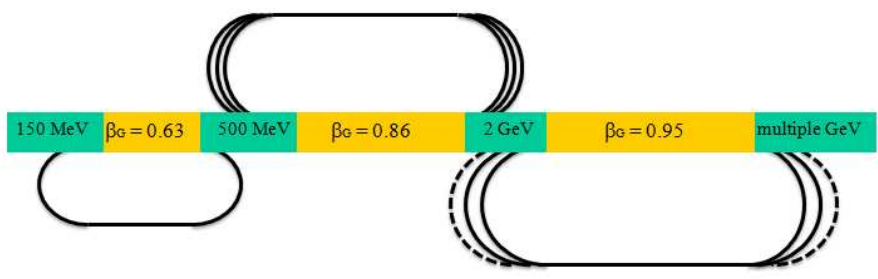

Figure 2: A schematic plot a $\mathrm{GeV}$ recirculating proton linac. 


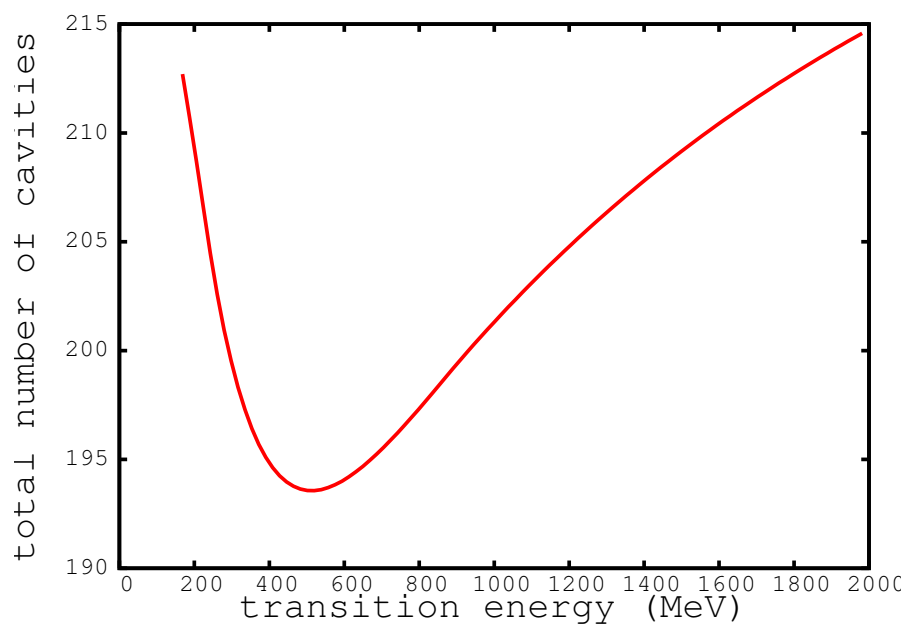

Figure 3: The total number of RF cavities in two sections as a function of the transition energy between the section one and the section two.

the energy, i.e. $\beta_{\text {in }}$ and $\beta_{\text {out }}$, one needs to maximize the $\bar{T}$ in order to minimize the number of cavities used. For the proposed energy range $(150 \mathrm{MeV}-2 \mathrm{GeV})$ in this study, we will use two accelerator sections, that is

$$
\Delta E_{1,2 \max }=q V_{1} N_{1} \bar{T}_{1}+q V_{2} N_{2} \bar{T}_{2}
$$

where $V_{1}$ and $V_{2}$ are the average accelerating voltage per cavity in each section, $N_{1}$ and $N_{2}$ are the average number of cavities in each section, and $\bar{T}_{1}$ and $\bar{T}_{2}$ are the average transit time factor in each section. In order to minimize the total number of cavities of the two sections, we would like to minimize the $N_{1}+N_{2}$ with respect to the transition energy, the $\beta_{G 1}$, and the $\beta_{G 2}$ subject to above energy constraint Eq. 5. Figure 3 shows the total number of RF cavities in these two sections as a function of transition energy between the section one and the section two. Here, we assume that the average accelerating voltage per cavity is $13 \mathrm{MV}$ and $V_{1}=V_{2}$. It is seen that a transition energy at $500 \mathrm{MeV}$ will result in a minimum total number of cavities. This transition energy is consistent with some proposed transition energies listed in Table 1. Figure 4 shows the average transit time factor as a function of geometric $\beta_{G}$ in the section one and the section two. The maximum normalized average transit 


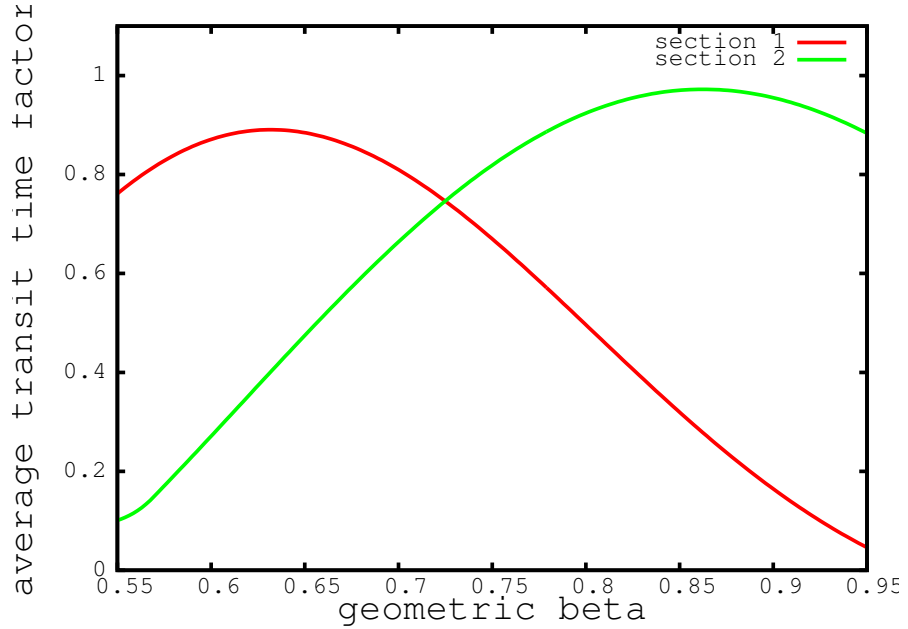

Figure 4: The average transit time factor as a function of geometric beta in the section one and the section two of the proposed accelerator.

time factor in section one is about 0.89 with a geometric $\beta_{G 1}=0.63$, and 0.97 with a geometric $\beta_{G 2}=0.86$ in section two. In the third section, beyond $2 \mathrm{GeV}$ energy, the change of proton velocity is small. We found that a 5-cell cavity with a geometric $\beta_{G 3}=0.95$ has close to 1 normalized average transit time factor for a wide range of final energy $(4-8 \mathrm{GeV})$.

The first section of the linac accelerates the proton beam from $150 \mathrm{MeV}$ to $500 \mathrm{MeV}$. In a single pass linac, it requires more than 34 cavities (assuming about $14 \mathrm{MV}$ per cavity accelerating voltage from a conservative consideration with off-crest acceleration). In the recirculating linac proposed here, the proton beam passes through those cavities multiple times. If we assume that the proton beam passes through this section of linac two times, this will reduce the number of cavities by half. The first pass of the proton beam exits from this section at $325 \mathrm{MeV}$ energy with a magnetic rigidity of $2.82 \mathrm{~T}-\mathrm{m}$. Assuming two Tesla bending magnets in the arc, this leads to a compact racetrack beam transport system with an arc radius of about one and a half meter. The second pass of the beam exits this section with $500 \mathrm{MeV}$ energy. This corresponds to a magnetic rigidity of $3.63 \mathrm{~T}-\mathrm{m}$. An achromatic dogleg lattice can be used to transport the beam into the second section. The second section accelerates the beam 
from $500 \mathrm{MeV}$ to $2 \mathrm{GeV}$. For a single pass linac, this requires more than 155 this will reduce the number of cavities in this section to 40, with $375 \mathrm{MeV}$ energy gain through each pass. The third section accelerates the beam from $2 \mathrm{GeV}$ to multiple $\mathrm{GeV}$ depending on the specific application. For an application such as the one proposed in the Project-X, this final energy can be $8 \mathrm{GeV}$. Assuming a to use minimum 300 cavities. On the other hand, using a recirculating linac in this section with multiple passes, e.g. six passes, the minimum number of cavities can be reduced to 50. Therefore, by using the multi-pass recirculating linac for the major energy range, the total number of the cavities needed in the accelerator can be reduced by more than a factor of four. Such a reduction of the accelerating cavity number in the linac significantly lowers the construction and operational cost of the facility. Moreover, the recirculating linac also shortens the length of the total straight accelerating section. This can be important if the facility is restricted by the available straight real estate.

In the recirculating linac, a proton beam passes through the same accelerating system multiple times to save the number of RF cavities. Due to the fact that the proton beam is still non-relativistic, the velocity of the beam changes during acceleration and varies from pass to pass (especially at lower energy section). This could cause a problem for multi-pass acceleration. When the RF cavity can be tuned to achieve a design phase (e.g. -30 degree) for the desired energy gain. When the beam passes through the accelerator for the second time, it has a higher energy and a larger velocity. Because the distance between accelerating cavities is fixed, within the same cavity, the proton will see 190 a different $\mathrm{RF}$ phase from that during the first pass. This will not be a problem if the driven phase of the cavity can be quickly adjusted between each pass or between each bunch train for CW operation so that the desired energy gain and design phase for the beam can be achieved. However, such a fast modulation of RF driven phase normally can not be easily attained for a superconducting 
Let $t_{i}^{m}$ denote the time pass between two RF cavities $i$ and $i+1$ during the $m^{t h}$ beam pass of the accelerator, $t_{i}^{n}$ the time pass between the two cavities during the $n^{t h}$ beam pass, if the difference of the two time pass satisfies the condition:

$$
t_{i}^{m}-t_{i}^{n}= \pm k T_{r f}, \quad k=0,1,2,3, \cdots
$$

where $T_{r f}$ is the oscillation period of the RF field inside the cavity, the proton beam will see the same phase of the cavity during multiple passes of the accelerator. For the first section two-pass recirculating linac proposed here, the above condition can be achieved through adjusting the distance between two cavities. Here, the above condition can be rewritten as:

$$
l_{i}\left(\frac{1}{\beta_{i}^{1}}-\frac{1}{\beta_{i}^{2}}\right)= \pm k c T_{r f}, \quad k=0,1,2,3, \cdots
$$

where $\beta_{i}^{1}=v_{i}^{1} / c$ is the normalized velocity after cavity $i$ during the first beam pass, $\beta_{i}^{2}=v_{i}^{2} / c$ the normalized velocity after cavity $i$ during the second beam pass, $c$ is the speed of light in vacuum, and $l_{i}$ is the distance between the cavity $i$ and the cavity $i+1$. Knowing the energy/velocity after each RF cavity during two beam passes, one can use above equation to determine the separation distance of two cavities to maintain the same RF phase.

Figure 5 shows the on-axis accelerating electric field profile for a $650 \mathrm{MHz}$ $\mathrm{RF}$ cavity with $\beta_{G}=0.63$. Assuming an on-axis peak accelerating gradient of $34 \mathrm{MV} / \mathrm{m}$ in the cavity and -30 degree design phase, one needs $34 \mathrm{RF}$ cavities to accelerate the proton beam from $150 \mathrm{MeV}$ to $500 \mathrm{MeV}$. If we assume the same RF phase for both beam passes, using above equation, we obtain the separation of two cavities shown in Fig. 6. It is seen that the first separation distance between the cavity 1 and the cavity 2 is slightly greater than the cavity length. The separation increases to about twice the cavity length at the end of the last two accelerating cavities (cavity 16 and 17). Figure 7 shows the evolution of 220 the proton kinetic energy through the total 17 cavities during the two beam passes with a fixed driven phase of each cavity. By appropriately arranging the 


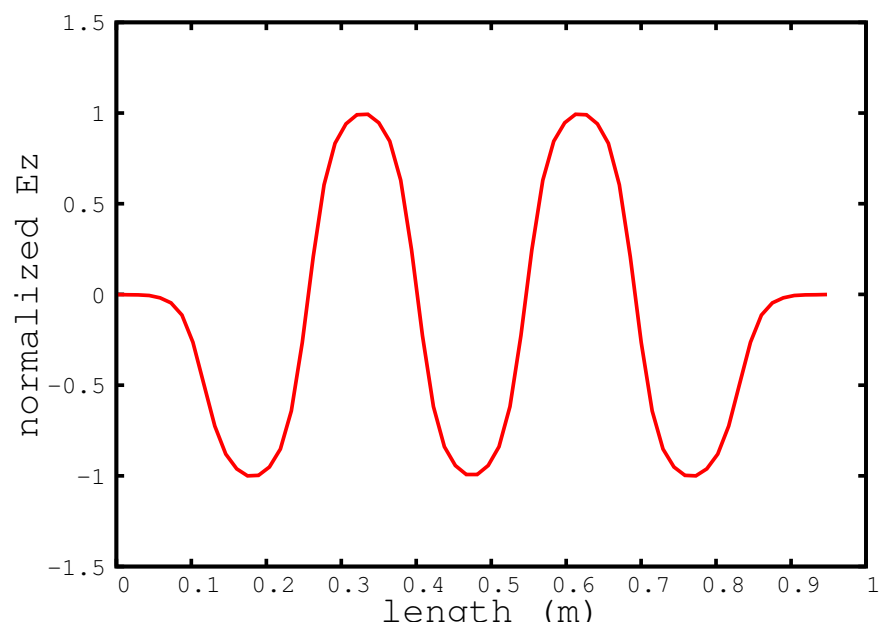

Figure 5: On-axis accelerating field profile of $650 \mathrm{MHz}$ cavity with $\beta_{G}=0.63$.

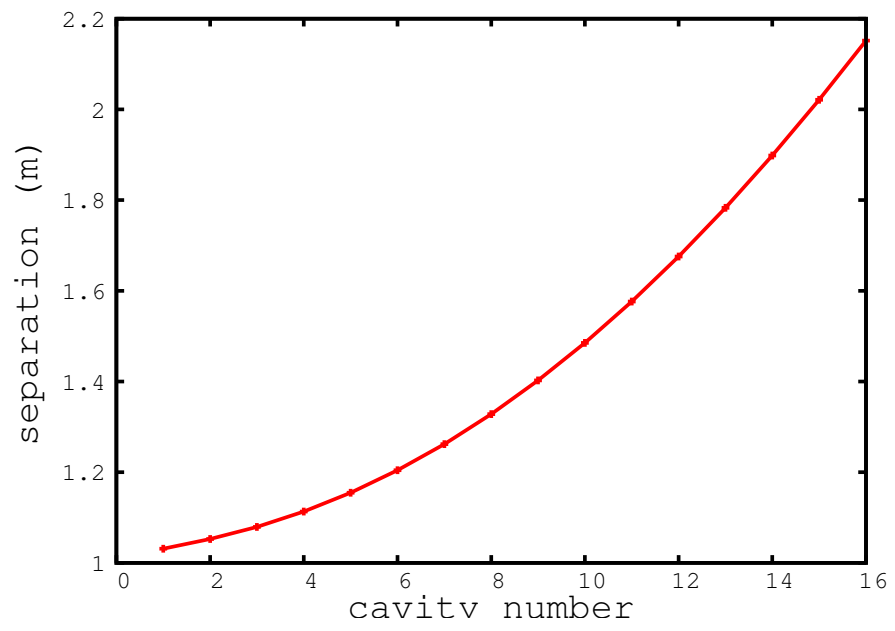

Figure 6: Separation distance between two cavities as a function of cavity number in the first section of the linac. 


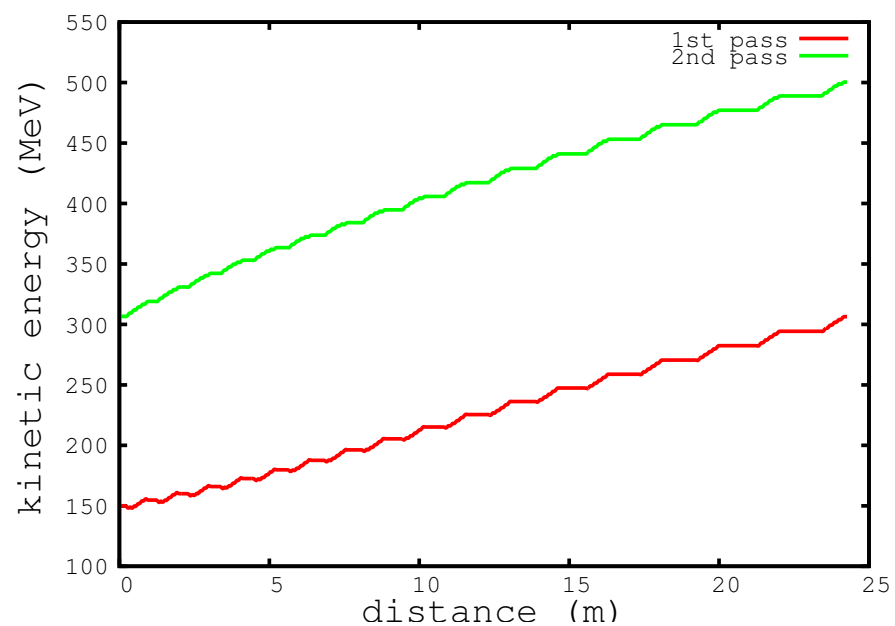

Figure 7: Two pass proton beam kinetic energy evolution through the first section of the recirculating linac.

separation distance of two cavities to satisfy the condition Eq. 6, the proton beam can be correctly accelerated with the designed RF phase in both passes.

Adjusting the distance between two cavities helps control RF phases during the two beam passes. However, for the multiple recirculating beam passes as proposed in section two and three, this will not work. Furthermore, as the proton beam energy increases, the velocity difference between two passes becomes smaller, this results in a very large separation distance in order to satisfy above condition. Another way to control the time delay (i.e. phase slippage) between multiple beam passes is to insert a phase shifter between two cavities. Such a phase shifter will control the time pass between two RF cavities for the multipass beam to either satisfy the condition Eq. 6 for the fixed RF design phases or some other RF design phases of each pass. A schematic plot of a section of accelerating cavities together with phase shifters is shown in Fig. 8, Magnetic 235 phase shifters have been used in free electron laser (FEL) light sources [25] and electron microtrons [26] to adjust the time delay of an electron beam. Using an achromat magnetic chicane as a phase shifter helps preserve beam quality after the shifter and also produces different time delay for different beam energies. A potential fast transverse kicker can be also added to the chicane for time delay 


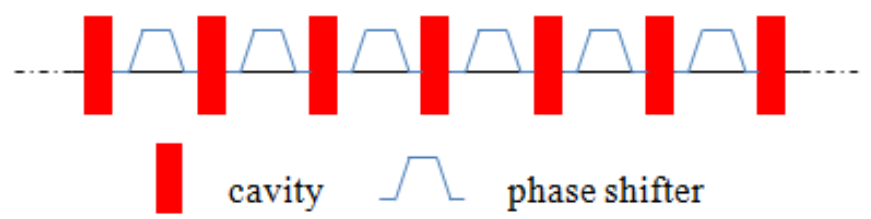

Figure 8: A schematic plot of a section of accelerating cavities with phase shifters.

240 this paper and will be reported in a future publication.

\section{Discussions}

A recirculating linac is a combination of the concept of a circular accelerator and a linear accelerator. It has some advantages of the circular accelerator by passing a beam through the same RF cavity multiple times to attain large energy gain. This saves the large number of RF cavities needed in a single pass linear accelerator and the corresponding construction and operational costs. Meanwhile, due to lacking of closed orbit of the circular accelerator, the recirculating linac can avoid some nonlinear resonances in the circular accelerator. This helps accelerate a high intensity beam to high energy while preserving the beam quality. The arc in the recirculating linac also provides a natural location for beam halo collimation.

On the other hand, the recirculating linac has a more complex accelerator architecture compared with the straight linear accelerator. It consists of not only a straight accelerating section but also arcs, merging, exiting, and straight transport systems. The same straight beam line will transport a beam with different energies. Proper design of the focusing lattice is needed to avoid significant beam mismatch in the accelerator. More advanced diagnostic system is also needed for this accelerator [24]. A protron beam traverses a longer path in the recirculating linac than that in the linear accelerator. The preservation of the beam quality to avoid transverse emittance growth and beam losses for high intensity beam with strong space-charge effects becomes more challenge. In the 
transport section without accelerating cavity, the proton beam may be subject to debunching and an increase of energy spread due to longitudinal space-charge effects. This could be compensated with an RF bunching cavity in the straight transport section. The transverse beam break-up instability might also be an issue in the recirculating linac. However, with a proper design of RF cavities, such an instability can be avoided.

Electron recirculating linacs have been under successful operation for many years. A lot of experience in the electron recirculating linac can be reused in the proton recirculating linac. One difference between these two linacs is that a proton beam has little coherent and incoherent synchrotron radiation through a bending magnet, which is beneficial to the preservation of beam quality. On the other hand, the space-charge effects are stronger in a proton beam than those 275 in an electron beam with the same energy and peak current. Special attention is needed to control those effects on the proton beam quality.

\section{ACKNOWLEDGEMENT}

We thank some useful discussions with Drs. J. Byrd and G. Huang about RF control. This research used computer resources at the National Energy Research Scientific Computing Center and was was supported by the U.S. Department of Energy under Contract No. DE-AC02-05CH11231.

\section{References}

[1] K. W. Jones and K. F. Schoenbert, "Operational status and future plans for the Los Alamos Neutron Science Center," Proc. of LINAC08, Victoria, BC, Canada, p. 88, 2008.

[2] G. Ciovati et al., "Superconducting prototype cavities for the Spallation Neutron Source (SNS) project," Proc. Particle Accelerator Conf., Chicago, IL, United States, 2001 (IEEE, New York, 2002), p. 484. 
[3] Y. Yamazaki et al., "Accelerator technical design report for J-PARC," KEK Report 2002-13, March 2003.

[4] J. Wei et al., "China spallation neutron source design," APAC 2007, Indore, India, p. 30 (2007).

[5] M. Eshraqi et al, "The ESS linac," in Proc. IPAC2014, Dresden, Germany, p. $3320(2014)$.

[6] H. Abderrahim et al., "Accelerator and target technology for accelerator driven transmutation and energy production," http://science.energy.gov/ media/hep/pdf/files/pdfs/ADS White Paper final.pdf (2010).

[7] Z. Li et al., Phys. Rev. ST Accel. Beams 16, 080101 (2013).

[8] R. Pande et al., Pramana - J. Phys. 78, 247 (2012).

[9] H. Ait Abderrahim et. al., Nuclear Physics News, vol. 20, no. 1, 2010, p. 24.

[10] G. P. Lawrence and T. P. Wangler, "Integrated normalconducting/superconducting high-power proton linac for the apt project," p. 1156 (1998).

[11] S. Holmes et al., "Project X Reference Design Report," Project Xdocument 776-v7, (2013).

[12] P. Ostroumov, New Journal of Physics 8, 281 (2006).

[13] F. Gerigk et al., "Conceptual design of the SPL II," CERN-2006-006, 2006.

[14] C.W. Leemann et al., "The Continuous Electron Beam Accelerator Facility: CEBAF at the Jefferson Laboratory," Annual Review of Nuclear and Particle Science 51 (2001), 413

[15] A. Richter, "Operational experience at the SDALINAC," EPAC 96, Sitges (1996) 110. 
[16] http://www.classe.cornell.edu/Research/ERL/

315 [17] J. N. Corlett et al., "LUX - a recirculating linac-based facility for ultrafast x-ray science," in Proc. of EPAC 2004, Lucern, Switzerland, p. 2439 (2004).

[18] K. Jordan et al., "JLAMP: an amplifier-based FEL in the JLab SRF ERL driver," JLAB-ACT-07-621, 2007.

[19] P. H. Williams et al., Phys. Rev. ST Accel. Beams 14, 050704 (2011).

${ }_{320}^{20}$ [20] R. C. York, Phys. Rev. ST Accel. Beams 17, 010705 (2014).

[21] http://www.lepp.cornell.edu/Events/ERL09/; ～http://erl2011.kek.jp/; http://ssrc.inp.nsk.su/conf/ERL2013/.

[22] S. A. Bogacz and V. A. Lebedev, Nucl. Instr. Meth. Phys. Res. A 503, 306 (2003).

325 [23] J. J. Barnard et al., Phys. Fluids B 5, 2698 (1993).

[24] D. Douglas, "An instrumentation wish list for high power/high brightness ERLs," Proceedings of Beam Instrumentation Workshop BIW10, Santa Fe, New Mexico, 506(2010).

[25] H. H. Lu, Y. Li, J. Pflueger, Nucl. Instr. Meth. Phys. A 605, p. 399 (2009).

330 [26] G. A. Novikov et al., Nucl. Instr. Meth. Phys. A 524, p. 60 (2004). 
figure 1

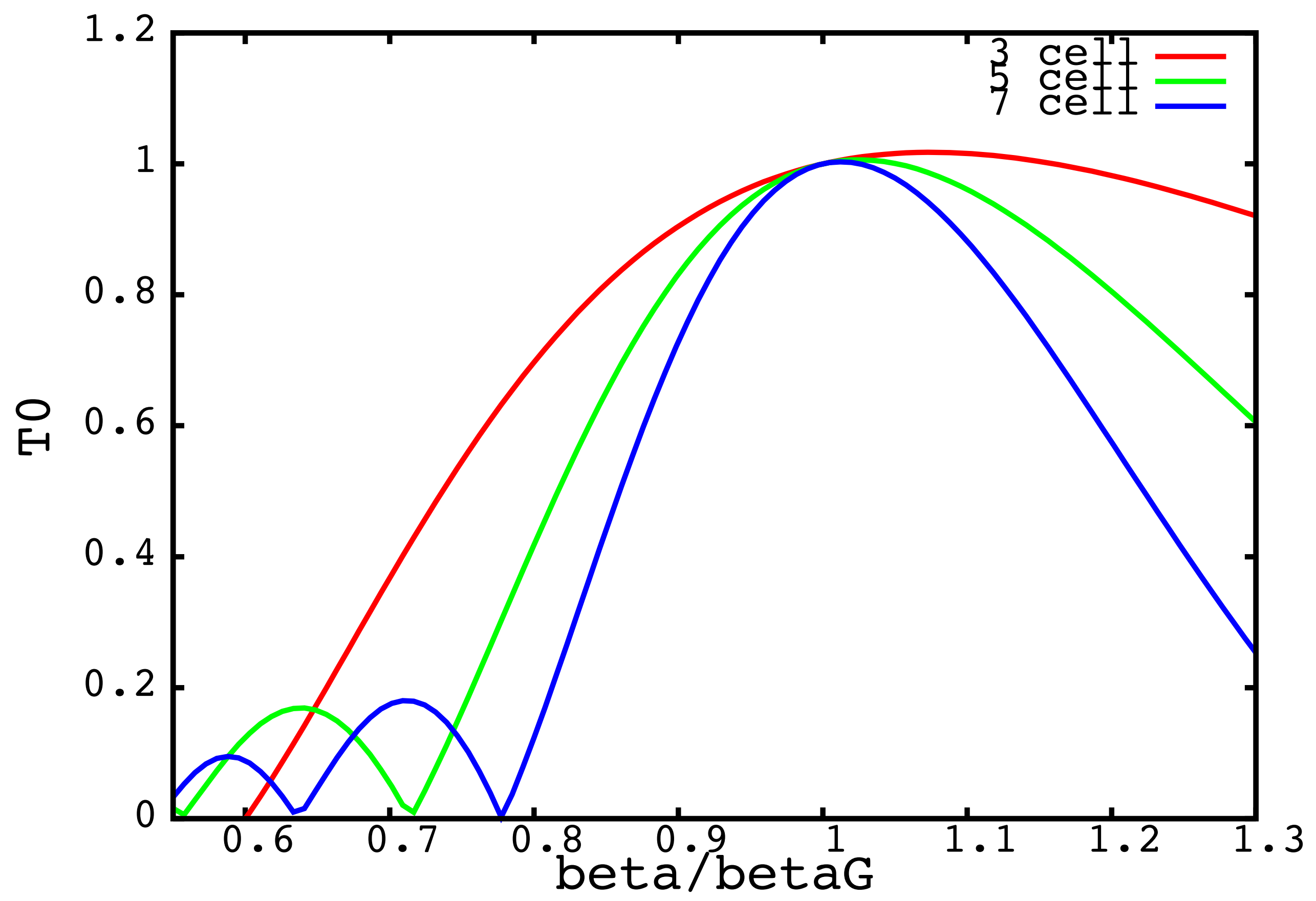


figure 3

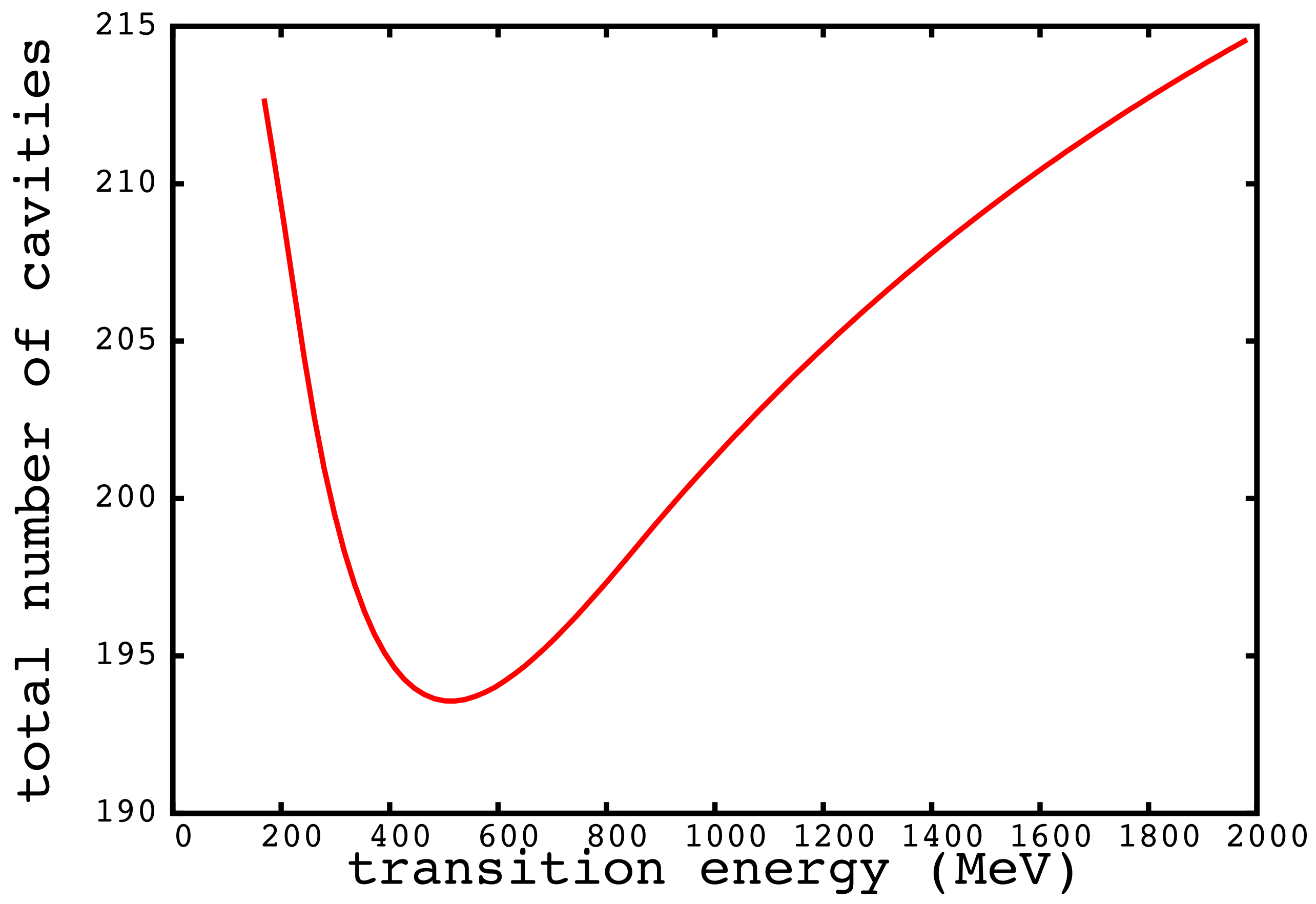


figure 4

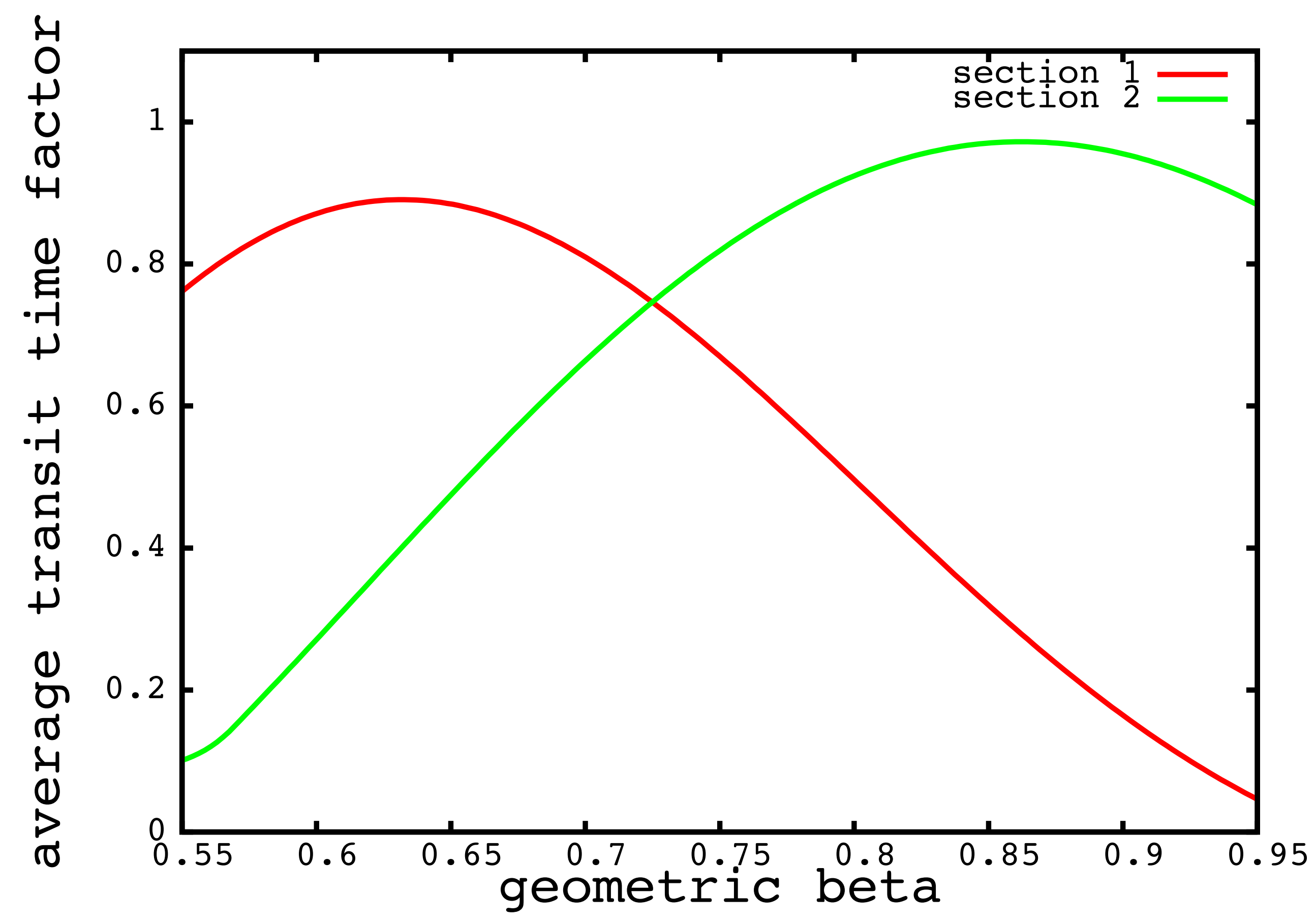


figure 5

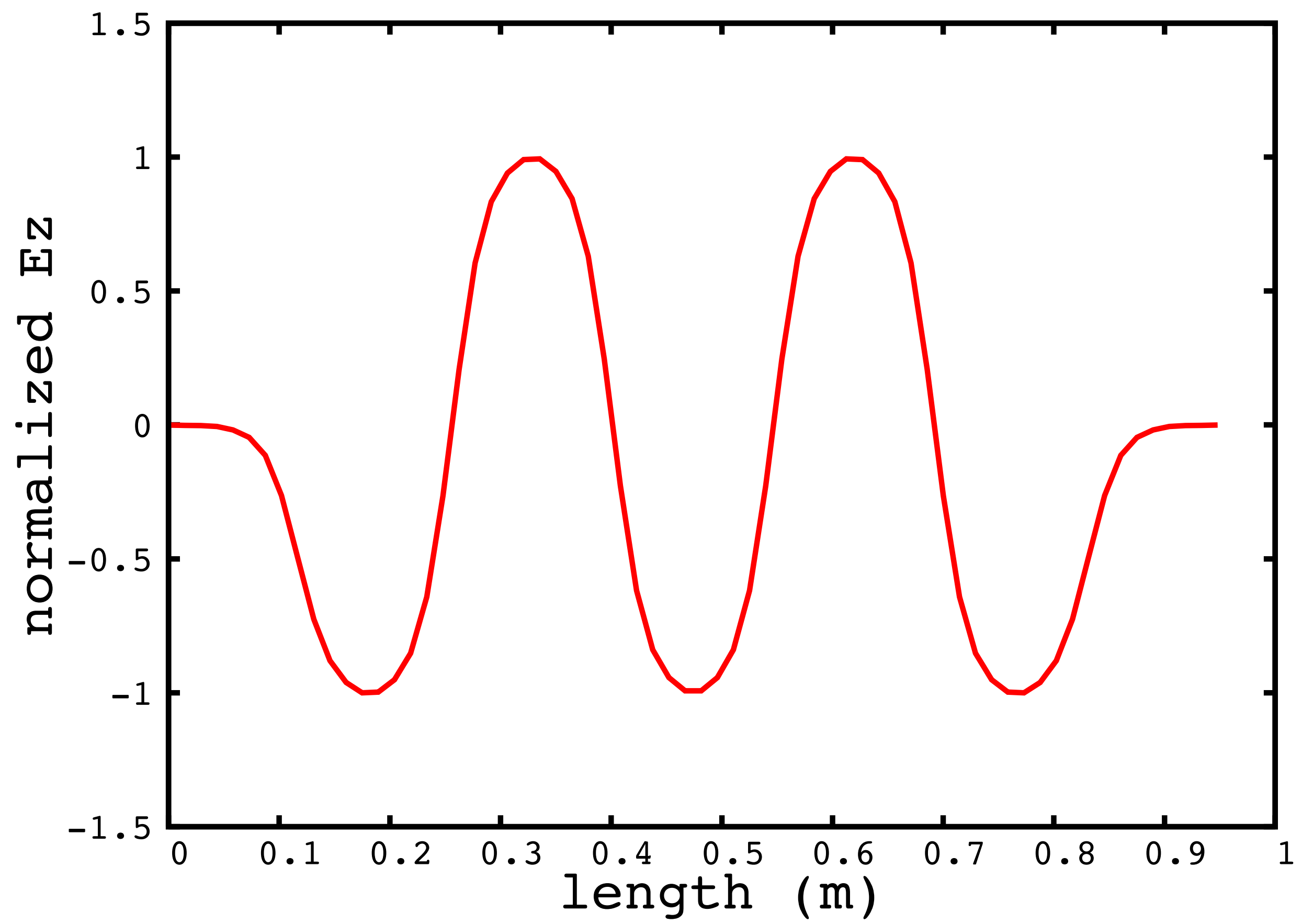




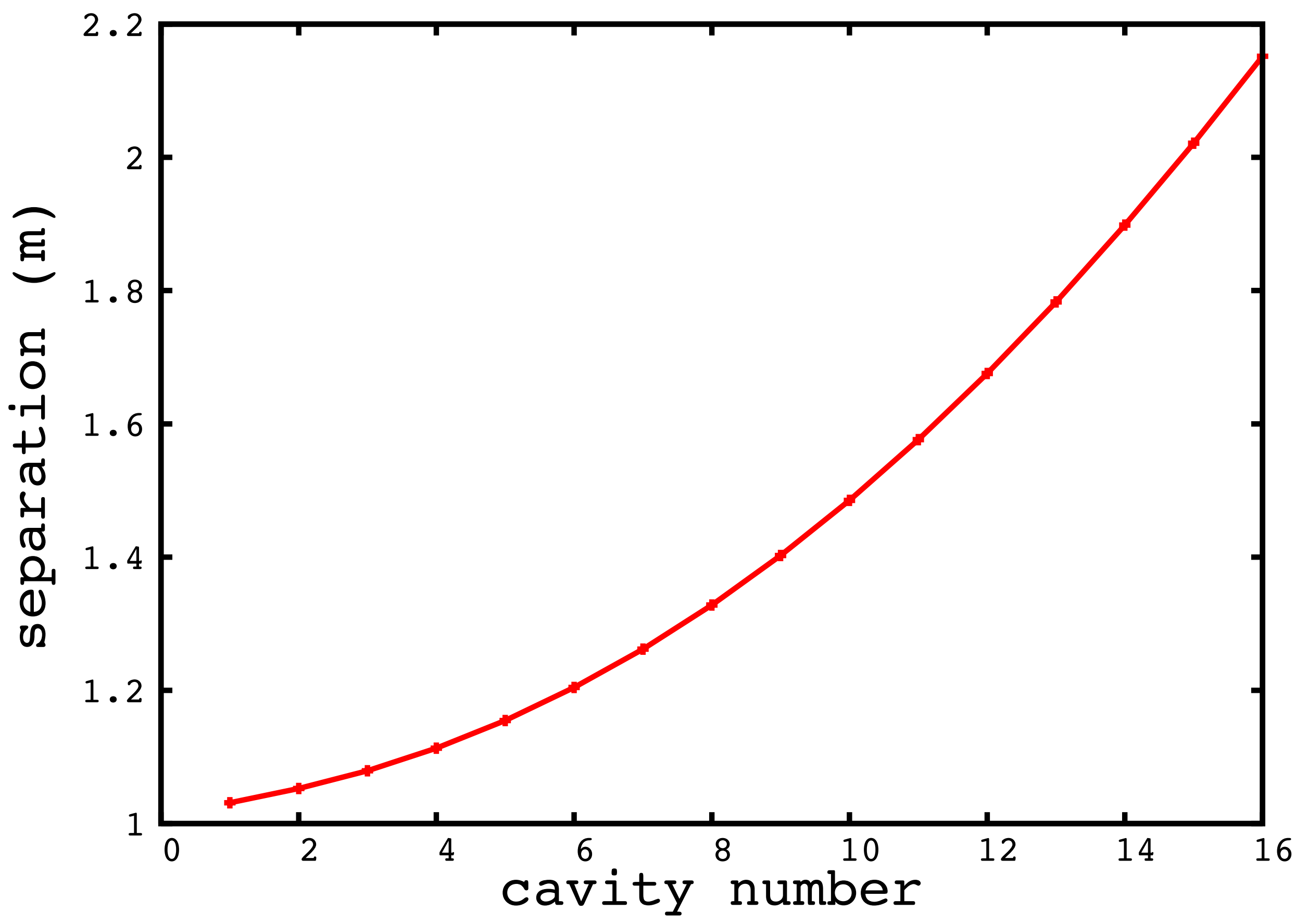


figure 7

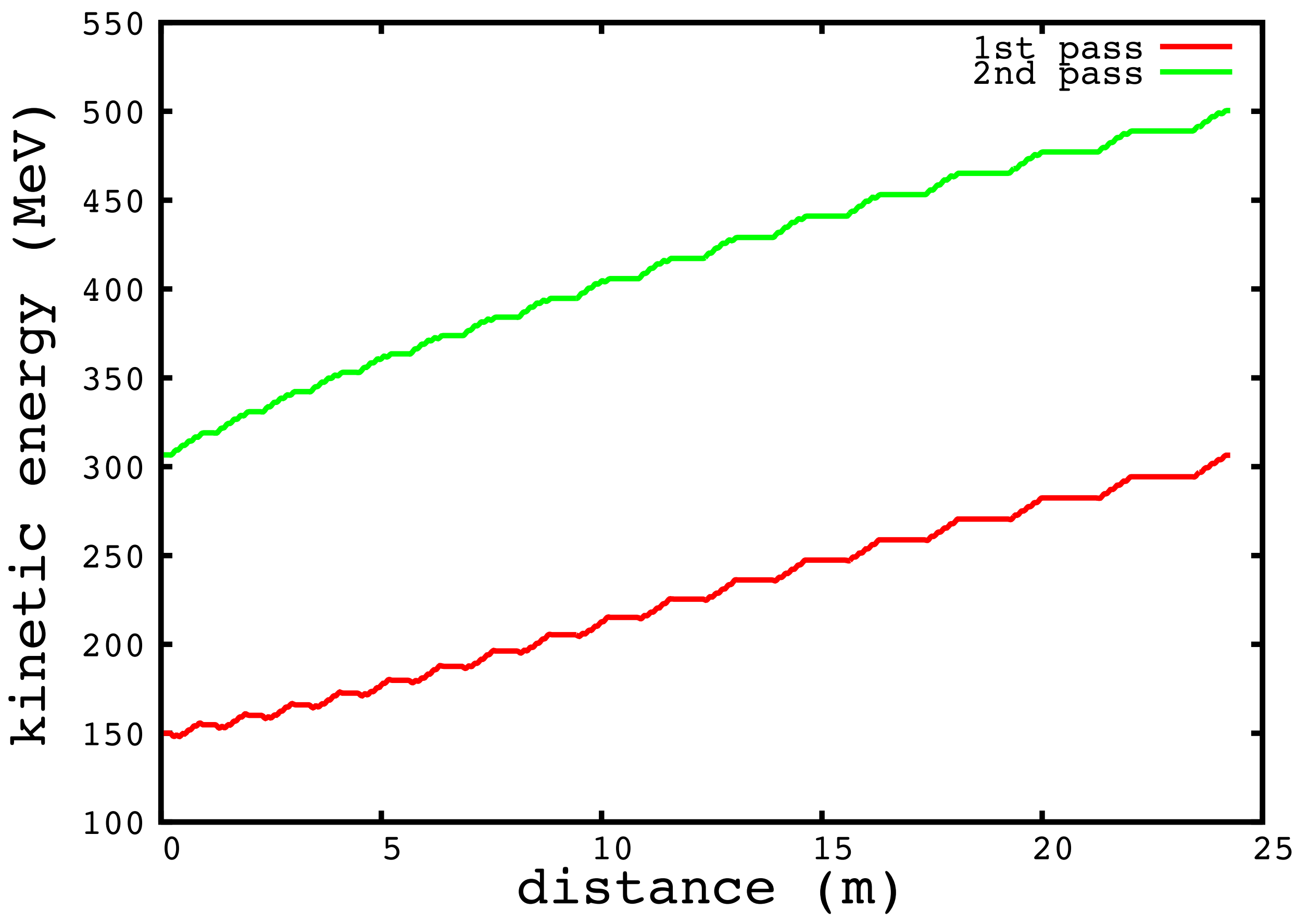


figure 8

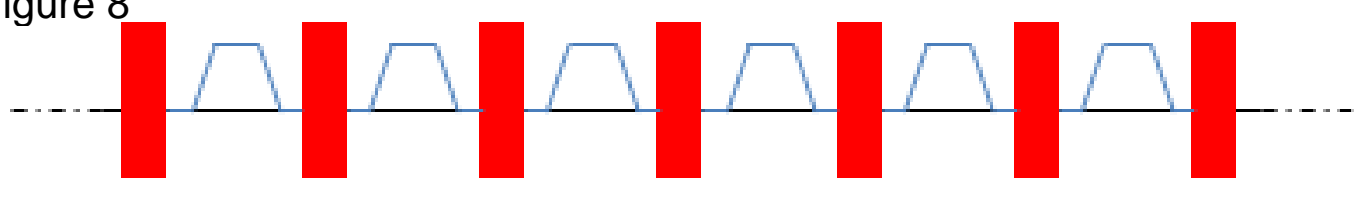
cavity $\_$phase shifter 